\title{
T cells fingered as culprits for narcolepsy
}

After nearly four decades, a Swiss-German collaboration of academic researchers and clinicians has been able to confirm long-held suspicions about the autoimmune origins of narcolepsy. Narcolepsy is a sleep-attack disorder that affects approximately 1 in 2,000 people in Europe and North America. The condition results from the loss of hypothalamic neurons that produce the protein hypocretin. What causes that neurodegeneration, however, was not known until the publication of two recent reports that identified the autoreactive $\mathrm{T}$ cells responsible for the neuronal loss, one in Nature $(\mathbf{5 6 2}, \mathbf{6 3 - 6 8 , 2 0 1 8 )}$ and a second on the bioRxiv preprint server (https:// doi.org/10.1101/378109, 2018).

Claudio Bassetti, a neurologist in the Department of Neurology at the University Hospital, Bern, and one of the authors on the Nature paper, says that until now evidence tying the deterioration of the hypocretinproducing neurons to autoimmune attack was "circumstantial." This is "the first observation of clear immune system involvement," he says.

That circumstantial evidence included the close association between the occurrence of narcolepsy-cataplexy and the presence of the HLA allele DQB1*0602, as well as immune dysregulation in narcolepsy. "The HLA connection has been known since the 1980s," says Bassetti, but pinning down the evidence for it required collaboration with research groups in basic immunology.

Immunologist Federica Sallusto at the ETH Zurich and the Institute for Research in Biomedicine at the University of Italian Switzerland in Bellinzona had developed a highly sensitive method for isolating

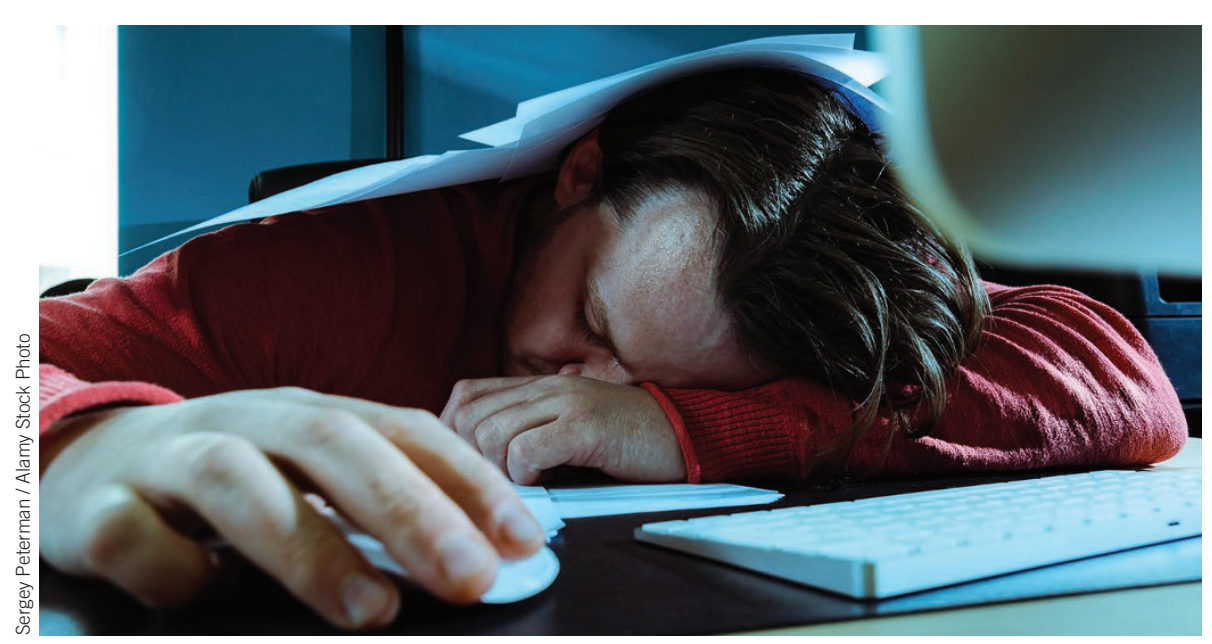

Immune cells targeting hypocretin-producing neurons have been found in people with the sleep disorder narcolepsy.

antigen-specific $\mathrm{T}$ cells in the human naive T-cell repertoire. The method expands memory $\mathrm{T}$ cells $\left(\mathrm{CD} 45 \mathrm{RA}^{-} \mathrm{CD} 4^{+}\right.$cells) polyclonally and then screens those expanded clones for proliferation in response to autologous B cells primed with antigen components.

Through the collaboration, Sallusto applied the approach to well-characterized narcolepsy clinical samples from patients across Switzerland. Specifically, they were looking for autoreactive T cells that could mount a response to hypocretin, potentially "a signature enabling early detection of the disease," as Bassetti put it.

One conventional, but indirect, way of diagnosing narcolepsy and monitoring its progression is to measure the levels of hypocretin in the cerebrospinal fluid. Hypocretin regulates wakefulness by, among other mechanisms, dampening serotonin production. The collaborators were looking for signs of autoimmune involvement.

Using blood and spinal fluid samples, the group looked for T-cell repertoires with the capacity to recognize hypocretin peptides and respond by proliferation. They compared the $\mathrm{T}$ cells of 19 patients with narcolepsy with those of 13 healthy donors who carried the narcolepsy-associated HLA allele DQB ${ }^{\star} 0602$. The patient group included 16 people with narcolepsy type 1 (NT1), characterized by less than 110 picograms hypocretin protein per milliliter cerebral spinal fluid, and 3 patients with the milder narcolepsy type 2 (NT2), showing normal or intermediate levels of hypocretin.

The results were clear. T cells from all patients with NT1 or NT2 except one showed a proliferative response to hypocretin. In contrast, the researchers found only a few proliferating T-cell lines among healthy controls. The frequency of hypocretin-reactive $\mathrm{T}$ cells was significantly higher in patients with NT1 or NT2 than in controls.

The researchers also found, in some cases, rare hypocretin-responsive cytotoxic $\mathrm{T}$ cells $\left(\mathrm{CD}^{+}\right.$cells). Because these cells can kill cells directly, their presence in patients with normal hypocretin levels in cerebrospinal fluid and no cataplexy, may indicate the phase in which neurons are destroyed. This could have have been the case for one individual with relatively high levels of $\mathrm{CD}^{+}$and $\mathrm{CD} 8^{+} \mathrm{T}$ cells against hypocretin, who was initially diagnosed with the milder NT2 form of narcolepsy but subsequently progressed to the more severe NT1.

That hypocretin-targeting immune cells are present in people with narcolepsy resolves a long-standing mystery, but the finding seems unlikely to have a major impact on clinical practice, at least in the short term. Bassetti believes that if the findings are confirmed, "there may be space for immunomodulatory intervention." He argues that there have been major advances in treating the symptoms of narcolepsy in the past 20 years and that immunomodulatory approaches could be applied if clinicians can identify patients in a transitional state in slowly progressive disease. However, he cautions that detecting autoimmune destruction post hoc would not help intervention in acute disease where neuronal loss is rapid.

Thomas Scammell, a neurologist in the Department of Neurology at the Beth Israel Deaconess Medical Center and Harvard Medical School, says that clinicians may not be surprised by this latest finding because the association between narcolepsy and the HLA allele DQB1*0602 was already well known. "Over $90 \%$ of narcolepsy patients carry 0602 , which strongly suggests that $\mathrm{T}$ cells are an important part of the mechanism that kills the hypocretin neurons," he says.

Scammell, who has provided consulting services to at least five pharmaceutical companies with narcolepsy therapies, also says that current industry interest in T-cell modulation in narcolepsy is low. "All the companies I consult with focus on the symptomatic features. None are putting energy in the immunological mechanism." But he does think that the confirmation of an autoimmune mechanism in narcolepsy may spark a change among some clinicians. "While the pharma companies are not yet interested on a T-cell mechanism, this research may spur clinicians to try T-cell modulation medications." he says.

Barbara Nasto Cambridge, UK 